\title{
Development of Prevention Strategies Against Bath- related Deaths Based on Epidemiological Surveys of Inquest Records: A Model Study in Kagoshima Prefecture
}

Midori Katsuyama

Kagoshima University

Eri Higo

Kagoshima University

Machiko Miyamoto

Kagoshima University

Takuma Nakamae

Kagoshima University

Daiko Onitsuka

Kagoshima University

Akiko Fukumoto

Kagoshima University

Masahiko Yatsushiro

Kagoshima University

Takahito Hayashi ( $\nabla$ takahito@m2.kufm.kagoshima-u.ac.jp )

Kagoshima University

\section{Research Article}

Keywords: bath-related death, Sudden death, environmental temperature, Alert system

Posted Date: April 26th, 2021

DOI: https://doi.org/10.21203/rs.3.rs-421814/v1

License: (c) (i) This work is licensed under a Creative Commons Attribution 4.0 International License. Read Full License 


\section{Abstract}

Sudden death in the bathroom (bath-related death) occurs more frequently in Japan than in other countries, especially among the elderly. To clarify the epidemiological characteristics of bath-related deaths, we reviewed inquest records of deaths in Kagoshima Prefecture from 2006 to 2019. We identified 2,689 cases of bath-related death. Of these cases, $90 \%$ were among people aged $\geq 65$ years. The majority of cases occurred in a home bathtub between 16:00 and 20:00. Most deaths (52.0\%) occurred in winter (December to February), and there was a significant negative correlation with the environmental temperature (maximum, minimum, and mean) on the day of death. We identified the environmental temperature that bath-related deaths were likely to occur in each region of Kagoshima. The causes of death were cardiac death in 1,231 cases and drowning in 855 cases. Forensic autopsies were performed in only 29 cases. Although autopsies are essential to elucidate the pathogenesis of bath-related deaths, it is difficult to increase the rate of autopsies under the current Japanese death investigation system. Therefore, we suggest that the best way to prevent bath-related death is establishing an "Alert system" based on our results, and to have people refrain from bathing on dangerous days.

\section{Introduction}

Sudden death in the bathroom (bath-related death) is much more common in Japan than in other countries, probably due to the unique style of bathing in Japan [1-10]. Some reports show that bathrelated deaths account for more than $10 \%$ of all sudden deaths, or approximately 14,000 cases per year in Japan [11]. The incidence rate is particularly high among people aged $\geq 65$ years, and is likely to increase as the population ages, making bath-related deaths a serious social problem.

In the 23 wards of Tokyo, Osaka city, and Kobe city, where administrative autopsies are performed on bath-related deaths under the medical examiner system, the three major causes of bath-related death are acute cardiac death (i.e., ischemic heart disease), drowning, and cerebrovascular accident [12]. However, forensic autopsies are not performed on bath-related deaths in many of the remaining prefectures that do not have the medical examiner system. In such prefectures, it is left to the discretion of police whether or not to conduct a forensic autopsy, and the decision is based more on evidence of criminal death rather than determining the cause of death. To prevent bath-related death, it is essential to elucidate the pathogenesis and mechanism of the death, and to investigate the cause of death through forensic autopsy. However, it is difficult to rapidly increase the number of autopsies of bath-related deaths under the current Japanese death investigation system as described above. Therefore, an urgent alternative is needed to prevent bath-related deaths.

According to previous literature, bath-related deaths occur overwhelmingly in colder temperatures, and our previous study found similar characteristics in the warmer Kagoshima Prefecture (annual mean air temperature in Kagoshima city is $19.2^{\circ} \mathrm{C}$ ), located in the southwest of Japan. Although the mechanism of bath-related death has not been elucidated, several reports suggest that some bath-related deaths are induced by the difference in temperatures between the low-air temperature in the bathroom and high- 
water temperature in the bath $[13,14]$. Such a temperature difference may cause wild fluctuations in blood pressure, resulting in arrhythmia, ischemic heart disease, stroke, and death, or loss of consciousness followed by drowning. Therefore, we conducted a retrospective survey of inquest records of bath-related deaths in Kagoshima Prefecture that occurred during 2006 to 2019 to determine the epidemiological characteristics of the deaths and to identify the environmental temperatures at which bath-related deaths were more likely to occur. Our final goal is to develop prevention strategies to avoid bath-related deaths.

\section{Materials And Methods}

All inquest records $(n=29,406)$ were collected for deaths during the period of 2006 to 2019 in Kagoshima Prefecture with the cooperation of the First Department of Criminal Investigation of the Kagoshima Prefectural Police Headquarters. We retrospectively reviewed the inquest records of cases that died unexpectedly in the bathroom while bathing, and extracted inquest records involving death that occurred in the bathroom, including death in spas and other bathing facilities. The records of sudden death in the bathroom were carefully gathered and assessed. In each case, we reviewed parameters, such as age, sex, date of incidents, place of death (home, spa, other bathing facilities), place of incidents in the bathroom (bathtub, space next to a bathtub, dressing room, sauna), living style (alone, with family), a history of alcohol use before bathing, a past history of illness, cause of death, and detail, of environmental temperature on the date of death (maximum, minimum, mean, temperature difference within a day), and the area where individual cases lived (subdivided into 19 regions based on the jurisdiction of the police station in Kagoshima Prefecture).

Statistical analysis was performed using Z-test under a binomial distribution of counts for comparisons between men and woman in total numbers and age-specific mortality rate. The Receiver Operating Curve (ROC curves) for the incidence of bath-related deaths at each environmental temperature was developed, and cut-off values for the temperature at which bath-related deaths are likely to occur were calculated (JMP Pro9, SAS Institute Inc., Cary, NC, USA). The correlation coefficient of each temperature and mortality rate was determined using Spearman's correlation coefficient by rank test. A value of $p<0.05$ was considered to indicate a significant difference.

This study was approved by the Ethics Committee for Epidemiological Research, Graduate School of Medical and Dental Sciences, Kagoshima University (Approval No. 200248) and was carried out in accordance with the Declaration of Helsinki Principles. Moreover, this study was conducted using inquest records from the past, and we could not obtain informed consent from the bereaved family for the use of the records. Therefore, in accordance with the "Ethical Guidelines for Medical Research Involving Human Subjects (enacted by the Ministry of Health, Labor and Welfare in Japan)," Section 12-1 (2) (a) (c), information on the implementation of the study was posted on our website (http://www.kufm.kagoshimau.ac.jp/ legalmed/), and if there was a request to refuse the use of the samples for research, they were excluded from samples of this study. In addition, the Ethics Committee for Epidemiological Research, 
Graduate School of Medical and Dental Sciences, Kagoshima University (Approval No. 200248) has approved the waiver for the informed consent of this study.

\section{Results}

A total of 2,689 cases of bath-related deaths (1,375 men and 1,314 women) were reported in Kagoshima Prefecture from 2006 to 2019 (crude yearly mortality rate, 8.9\% to $12.7 \%$ per 100,000 individuals). No significant difference was observed in the total number of bath-related death between men and women $(p=0.238)$, however, there were significantly higher men than women for cases $>60$ years of age $(p=0.0446),>80$ years of age $(p=0.0239)$, and $>90$ years of age $(p=0.0294)$. The mean age of the 2,689 cases was 77.9 years, (range, 4 to 103 years). Of the 2,689 cases of bath-related death, 2,419 cases $(90.0 \%)$ were $\geq 65$ years old (Fig. 1A), and the mortality rate of bath-related death increased with age (Fig. 1B).

Most of the cases of bath-related death occurred at home $(n=2,292 ; 85.2 \%)$, followed by spas and other bathing facilities including hospitals, nursing homes, and hotels (Table $1 \mathrm{~A}$ ). In the bathroom, the bathtub was the most frequent location, occurring in 2,427 cases (90.3\%), followed by the space next to a bathtub in the bathroom and the dressing room (Table 1B). Most deaths were recorded between 16:00 and 20:00 (44.0\%), a time when the elderly usually take a bath in Japan (Fig. 2).

As for living style, of the 2,689 cases of bath-related death, 1,048 (45.9\%) of the cases lived alone and 1,237 cases (54.1\%) lived with their family. In Kagoshima Prefecture, there were 257,593 people who lived alone and 1,401,304 people who lived with their family. Therefore, those who lived alone had a substantially higher mortality rate of bath-related death compared with those who lived with their family (406.8 vs. 88.3 individuals per million). In addition, we compared the postmortem interval until discovery for these two groups (Fig. 3). For cases who lived with their family, it took less than 2 hours on average until being discovered, whereas, for cases who lived alone, it took substantially longer time to be discovered. In addition, there was no significant gender difference in the time required for discovery (data not shown).

A history of alcohol drinking before bathing was reported in only $115(4.3 \%)$ of the deaths (men 97; women 18). However, in 730 cases $(27.1 \%)$, it was not known whether they had been drinking alcohol before bathing. A past history of illness with incidence is presented in Table 2. Two hundred forty-two cases $(9.0 \%)$ were considered to be healthy and the remaining 2,447 cases had some past history of illness, including hypertension, cardiovascular disease, diabetes mellitus and central nervous system disorder. The most common illness was hypertension ( 1,213 cases; $45.1 \%)$, in combination with other illnesses in most cases.

Surprisingly, forensic autopsies were performed in only $29(1.08 \%)$ of the deaths. Of these 29 cases, the most common cause of death was drowning ( 24 cases, $82.8 \%$ ), followed by ischemic heart disease ( 2 cases, $6.9 \%)$. In the remaining 3 cases, the cause of death was diagnosed as unknown due to advanced decomposition. Of the 24 cases diagnosed as drowning, the cause of drowning was identified in 9 cases, 
while the cause of drowning was unknown in the remaining 15 cases. The most common cause of drowning was cardiovascular disease (3 out of 9 cases), and other causes included metabolic disease such as liver cirrhosis, epilepsy, chronic subdural hematoma, and obstructive sleep apnea syndrome. In the other 2,660 cases, the cause of death was determined only by external examination with or without postmortem CT. Figure 4 demonstrates that the cause of death indicated on the death certificate was heart disease in 1,231 cases (45.8\%), drowning in 855 cases (31.8\%), and central nervous system disorders in 340 cases $(12.6 \%)$.

There was a clear seasonal trend in the occurrence of bath-related death (Fig. 5A). The greatest number of deaths occurred during winter months (1,399 cases, $52.0 \%$ in December to February), whereas the summer months (June to August) had the least number of deaths (215 cases, 8.0\%). There was no significant difference in the seasonal trend with gender. Next, a study was conducted to identify the environmental temperatures (maximum, minimum, mean, temperature difference within a day) at which the bath-related deaths occurred. Since Kagoshima Prefecture is extends a significant distance from north to south (approximately $600 \mathrm{~km}$ ), including some remote islands, and the environmental temperature varies from region to region, we divided the Prefecture into 19 regions according to the jurisdiction of the police station that handles corpses (Fig. 5B). Among the environmental temperatures, the maximum, minimum, and mean temperatures all showed significant negative correlations with the incidence of bath-related deaths (Suppl. Table 1). However, the temperature at which bath-related death were more likely to occur differed by region (maximum, 16-24 (median 21.5) ${ }^{\circ} \mathrm{C}$; minimum, 6.5-18.5 (median 11) ${ }^{\circ} \mathrm{C}$; mean, $10-21$ (median 16) ${ }^{\circ} \mathrm{C}$ ) (Table 3). In particular, in the remote islands located in the south of Kagoshima Prefecture (O, P, Q, R, S in Fig. 5B), the temperatures at which bath-related death occurred tended to be higher than those in other regions. On the other hand, temperature difference within a day showed a significant positive correlation with the incidence of the death in only 6 regions $(A, H, I, J$, $L, Q$ ), but not in the remaining regions. The temperature differences within a day in the 6 regions where bath-related deaths are likely to occur were calculated to be 7.5-14 (median 9.25) ${ }^{\circ} \mathrm{C}$ or more.

\section{Discussion}

During 2006-2019, there were 29,406 inquest cases in Kagoshima Prefecture. Among them, 2,689 (9.1\%) were cases of bath-related death. The number of bath-related deaths was approximately 2.8 times the number of automobile accidental deaths, and, therefore, bath-related death in Kagoshima Prefecture must comprise a relatively large percentage of all deaths. Moreover, the crude mortality rate (per 100,000 population) of bath-related death in Kagoshima Prefecture is estimated to be 8.9-13.4 (mean 11.4). This rate is similar to that in other prefectures of Japan including one in the north (8.3-10.0) [1-7]. On the other hand, the crude mortality rate of bath-related death in the United States (U.S.) is reported to be 0.16 [15], and the rate in Kagoshima Prefecture is approximately 61-fold higher. In addition, the age groups in which bath-related death occur are clearly different between the U.S. and Kagoshima Prefecture. Bath-related death is most common among children $<5$ years old in the U.S. [15], with most deaths occurring as accidental drowning while playing in the bathtub. However, our results show that bath-related death is 
most common among those aged $\geq 65$ years ( $90 \%$ of all deaths) in Kagoshima Prefecture, with most deaths attributed to sudden natural causes such as ischemic heart disease. Accordingly, since the causes of bath-related death are fundamentally different between the U.S. and Japan, prevention strategies should be developed that are specific to Japan to reduce the number of bath-related deaths in Japan.

Our results indicate that bath-related death occurs most frequently in those aged $>65$ years and during the cold winter season, which is consistent with results reported previously from other prefectures in Japan [1-7]. Many bathrooms and other rooms in Japan are unheated, even in winter, and the cold temperatures have been considered to contribute to bath-related death [8]. The Japanese bathing style is to immerse the body in hot water $\left(>41^{\circ} \mathrm{C}\right)$ and elderly persons in Japan take a bath almost every day, especially in the winter [9]. Studies have indicated that patients diagnosed with angina pectoris may show electrocardiogram (ECG) changes that indicate ischemic changes or arrhythmias, such as ventricular tachycardia, supraventricular extrasystole, ventricular extrasystole, and/or a tendency of bradycardia, during usual bathing $[7,10]$. These arrhythmias, in particular ventricular tachycardia, may trigger a fatal event while in the bathtub. Thus, elderly people in Japan may have some undiagnosed underlying diseases that are risk factors of bath-related death.

In the present study, hypertension, a significant risk factor for cardiovascular mortality, was the most common past health issue among bath-related death cases. Several physiological studies have demonstrated that elderly people with hypertension often show a sudden decrease of blood pressure, followed by cardiopulmonary arrest $[1,5]$. The results of those studies, as well as our results suggest that cardiac events may occur more frequently in people with hypertension than in healthy individuals during bathing.

Our findings also indicate that most bath-related death occurs from 16:00 to 20:00, a time the elderly usually bathe in Japan, with most deaths occurring in the home bathroom. While alcohol consumption prior to bathing is a risk factor for bath-related death, only $4.3 \%$ (115 of 2,689 cases) of our cases of bathrelated death had consumed alcohol prior to taking a bath $[4,5,16,17]$. Therefore, bath-related death occurs frequently in the elderly without alcohol consumption.

Our results showed that the incidence of bath-related deaths was overwhelmingly higher among those living alone than among those living with their families. People who live alone take baths in a cold bathroom where nobody has bathed before them. In addition, there is no one who can help them when they have serious accidents while bathing. From a preventive perspective, elderly people, especially those with risk factors such as cardiac disease or hypertension should live with their family if possible. Even if they cannot live together, the elderly should be supervised by their neighbors and health visitors. In addition, the installation of remote monitoring systems should be considered, if possible. Recently, there have been engineering attempts in the bathroom to prevent bath-related deaths. For instance, a system has been devised to prevent drowning in the bathtub by installing a device on the bathroom wall that monitors ECG and respiratory status [18], and interlocking it with a relief device (such as automatically 
draining the water in the bathtub) when aforementioned dangerous change in ECG or respiratory status that could lead to a bathing accident is detected.

As expected, very few of the bathing deaths were subjected to forensic autopsy (29 cases, $1.08 \%$ ). Most of the deaths in the autopsy cases were due to drowning, and the distribution of causes of death was different from that in the cases without autopsy. In 9 of the 24 cases diagnosed as drowning, the cause of death could be determined by autopsy, and the most common cause was cardiovascular disease. According to a study of autopsy cases in the 23 wards of Tokyo where there is a medical examiner system, more than half $(54.5 \%)$ of the bath-related deaths had pathological findings indicating cardiovascular disease, but $18.4 \%$ had no findings (pathological findings, medicinal or toxic substances) that could lead to the cause of death [12]. Bath-related deaths are thought to be caused by a number of factors, and forensic autopsy is essential to determine the cause of death. Therefore, it is necessary to change the Japanese death investigation system from the current police-oriented system to a forensic pathologist-oriented system that truly emphasizes the investigation of the cause of death.

Our study demonstrates that environmental temperatures such as the maximum, minimum, and mean temperatures all showed significant negative correlations with the incidence of bath-related deaths. The temperature at which bath-related death was more likely to occur differed by region (maximum, 16-24 (median 21.5) ${ }^{\circ} \mathrm{C}$; minimum, 6.5-18.5 (median 11) ${ }^{\circ} \mathrm{C}$; mean, 10-21 (median 16) ${ }^{\circ} \mathrm{C}$ ). On the other hand, the temperature difference within a day showed a significant positive correlation with the incidence of the death only in 6 regions. The reason for the lack of correlation in other regions may be that bath-related deaths occurred when other environmental temperatures (maximum, minimum, and mean temperatures) were lower than those calculated above, even if the temperature difference within a day was not large. Therefore, we believe that bath-related deaths can be prevented by issuing warnings (so-called "BathRelated Death Alerts") to refrain from bathing on days when all environmental temperature conditions are met. The means of issuing warnings include our website, television, radio, and internet news. Since the daily maximum and minimum temperatures are known by 16:00 to 20:00, when Japanese people usually bathe, effective alerts could be issued. The best prevention strategy against bath-related deaths is to avoid bathing itself on dangerous days. If it can be confirmed that the number of bathing deaths can be reduced through the use of alerts for bath-related deaths in Kagoshima Prefecture, it may be possible to use artificial intelligence to analyze big data on bath-related deaths nationwide.

In conclusion, it is highly likely that bath-related deaths are closely related to the Japanese unique bathing culture. In addition, multiple factors are involved in bath-related deaths, and forensic autopsies are essential in order to elucidate the pathogenesis and mechanism of the death. However, the current Japanese death investigation system makes it difficult to increase the number of autopsies on bathrelated deaths rapidly, so the first priority is to reduce the number of bath-related deaths in Kagoshima Prefecture by issuing alerts based on the results of this study. If the alert is successful, Kagoshima Prefecture could be used as a model case for nationwide implementation.

\section{Declarations}




\section{Funding}

This work was supported by JSPS KAKENHI Grant Number JP18K10133.

\section{Conflicts of Interest}

All authors declare no conflict of interest related to this study.

\section{Acknowledgment}

We sincerely thank the staff of the First Department of Criminal Investigation of Kagoshima Prefectural Police Headquarters for their kind cooperation.

\section{Author Contributions}

M.K., E.H. and M.M. conceived and designed the research; T.N., D.O., A.F. and M.Y. prepared and analyzed data.; E.H. and M.M. prepared all figures and tables.; T.H. advised whole research.; M.K. and T.H. wrote the manuscript; All authors reviewed the manuscript and approved the final version of the manuscript for submission.

\section{Data availability}

The data that support the findings of this study are available from the corresponding author upon reasonable request.

\section{References}

1. Yoshioka, N., Nibe, H. \& Enzan, K. Sudden death in the bathroom and its medico-legal issues. Pract. Forensic Med. 41, 353-9 (1998). (Article in Japanese)

2. Kurosaki, K., Kuriiwa, F. \& Endo, T. Questionable diagnoses on the cases of sudden death while bathing. Pract. Forensic Med. 45, 175-80 (2002). (Article in Japanese)

3. Takahashi, N., Saitou, M. Sudden death in bath tub: Review of 56 cases in Naruko area. Pract. Forensic Med. 37, 391-5 (1994). (Article in Japanese)

4. Funayama, M., Yamaguchi, Y. \& Matuo, Y. Death related to bath. Pract. Forensic Med. 32, 301-7 (1989). (Article in Japanese)

5. Inamura, K. Sudden death of aged persons in bath tub. Pract. Forensic Med. 38, 349-51 (1995). (Article in Japanese)

6. Yoshioka, N., Chiba, T. \& Yoshizaki, K. Forensic consideration of death in the bathtub. Legal Med. 1, 375-81 (2003).

7. Chiba, T., Yamauchi, M. \& Yoshioka, N. Risk factors of sudden death in the Japanese hot bath in the senior population. Forensic Sci. Int. 149, 151-8 (2005).

8. Tochihara, Y. Bathing in Japan: A review. Hum. Environ. Syst. 3, 27-34 (1999). 
9. Takasaki, Y., Ohnaka, T. \& Yoshitake, S. Environmental and Behavioral conditions of bathing among elderly Japanese. Physiol. Anthropol. 26, 235-40 (2007).

10. Igarashi, T. Electrocardiographic changes during bathing in patients with angina pectoris by bathing Holter electrographic monitoring. JJCDP. 38, 16-25 (2003). (Article in Japanese)

11. Hori, S., Suzuki, M. \& Kurihara, T. Accidents during bathing. Nihon Rinsho 71, 1047-1052 (2013). (Article in Japanese)

12. Suzuki, H., Hikiji, W. \& Fukunaga, T. Characteristics of sudden bath-related death investigated by medical examiners in Tokyo, Japan. J Epidemio/ 25, 126-132 (2015).

13. Suzuki, M., Ikaga, T., Hori, S. Relationship between Bath-related Deaths and Low Air Temperature. Intern Med 56, 3173-3177 (2017).

14. Tahara, Y. Association Between Bathing and Sudden Death. Circ J 81, 1096-1097 (2017).

15. Budnick, L. D., Ross, D. A. Bathtub-related drownings in the United States, 1979-81. Am J Public Health 75, 630-633, doi:10.2105/ajph.75.6.630 (1985).

16. Kenttämies, A., Karkola, K. Death in sauna. J Forensic Sci 53, 724-729 (2008).

17. Yang, K., Choi, B. H. \& Yoo, S. H. Bath-related Deaths in Korea between 2008-2015. J Korean Med Sci 33, e108, doi:10.3346/jkms.2018.33.e108 (2018).

18. Motoi, K., Yamakoshi, Y. \& Yamakoshi, K. I. Measurement of electrocardiograms in a bath through tap water utilizing capacitive coupling electrodes placed outside the bathtub wall. Biomed Eng Online. Jan 11;16(1):12. doi: 10.1186/s12938-016-0304-9 (2017).

\section{Tables}

Table 1. Location where bath-related deaths occurred

A) Types of bathroom

\begin{tabular}{|llll|}
\hline & Male & Female & Total \\
\hline Home & 1,067 & 1,225 & $2,292(85.2 \%)$ \\
\cline { 2 - 4 } Spa & 246 & 54 & $300(11.2 \%)$ \\
\cline { 2 - 4 } Other & 62 & 35 & $97(3.6 \%)$ \\
& 1,375 & 1,314 & 2,689 \\
\hline
\end{tabular}

B) Location in the bathroom 


\begin{tabular}{|llll|}
\hline & Male & Female & Total \\
\hline Bathtub & 1,239 & 1,188 & $2,427(90.3 \%)$ \\
\cline { 2 - 4 } Space next to a bathtub & 108 & 112 & $220(8.2 \%)$ \\
\cline { 2 - 4 } Dressing room & 10 & 11 & $21(0.8 \%)$ \\
\hline Other & 18 & 3 & $21(0.8 \%)$ \\
\hline & 1,375 & 1,314 & 2,689 \\
\hline
\end{tabular}

Table 2. Past history of illness

\begin{tabular}{|llll|}
\hline & Male & Female & Total \\
\hline Hypertension & 555 & 658 & $1,213(45.1 \%)$ \\
\hline Cardiovascular diseases & 329 & 348 & $677(25.2 \%)$ \\
\hline Diabetes mellitus & 334 & 204 & $538(20.0 \%)$ \\
\hline CNS disorder & 277 & 207 & $484(18.0 \%)$ \\
\hline Cancer & 179 & 79 & $258(9.6 \%)$ \\
\hline Epilepsy & 28 & 24 & $52(1.9 \%)$ \\
\hline Others & 754 & 775 & $1,529(56.9 \%)$ \\
\hline No history (healthy) & 138 & 104 & $242(9.0 \%)$ \\
\hline
\end{tabular}

CNS, central nervous system

Table 3. Relation between environmental temperature and bath-related death in each Kagoshima region 


\begin{tabular}{|c|c|c|c|c|c|c|c|c|c|}
\hline \multirow[b]{2}{*}{ City } & \multirow[b]{2}{*}{$\mathrm{n}$} & \multicolumn{2}{|c|}{ Max temperature } & \multicolumn{2}{|c|}{ Min temperature } & \multicolumn{2}{|c|}{ Mean temperature } & \multicolumn{2}{|c|}{$\begin{array}{l}\text { Temperature } \\
\text { difference within a day }\end{array}$} \\
\hline & & ${ }^{\circ} \mathrm{C}$ & $p$ & ${ }^{\circ} \mathrm{C}$ & $p$ & ${ }^{\circ} \mathrm{C}$ & $p$ & ${ }^{\circ} \mathrm{C}$ & $p$ \\
\hline $\mathrm{A}$ & 580 & 20.5 & $<0.0001$ & 13.0 & $<0.0001$ & 17.5 & $<0.0001$ & 9.0 & $<0.0001$ \\
\hline B & 151 & 16.0 & $<0.0001$ & 11.0 & $<0.0001$ & 15.0 & $<0.0001$ & 9.5 & 0.5306 \\
\hline $\mathrm{C}$ & 44 & 22.0 & $<0.0001$ & 9.0 & $<0.0001$ & 16.0 & $<0.0001$ & 12.0 & 0.0905 \\
\hline $\mathrm{D}$ & 71 & 22.5 & $<0.0001$ & 11.0 & $<0.0001$ & 15.0 & $<0.0001$ & 8.0 & 0.0579 \\
\hline $\mathrm{E}$ & 129 & 17.0 & $<0.0001$ & 10.5 & $<0.0001$ & 15.0 & $<0.0001$ & 13.0 & 0.1994 \\
\hline $\mathrm{F}$ & 140 & 21.5 & $<0.0001$ & 11.0 & $<0.0001$ & 10.0 & $<0.0001$ & 10.0 & 0.2175 \\
\hline G & 51 & 21.0 & 0.0006 & 9.5 & $<0.0001$ & 16.0 & $<0.0001$ & 11.5 & 0.0722 \\
\hline $\mathrm{H}$ & 134 & 19.0 & $<0.0001$ & 10.5 & $<0.0001$ & 15.5 & $<0.0001$ & 9.0 & 0.0024 \\
\hline I & 117 & 21.0 & $<0.0001$ & 7.5 & $<0.0001$ & 15.5 & $<0.0001$ & 10.5 & 0.0264 \\
\hline $\mathrm{J}$ & 245 & 20.0 & $<0.0001$ & 9.0 & $<0.0001$ & 17.0 & $<0.0001$ & 14.0 & 0.0042 \\
\hline $\mathrm{K}$ & 54 & 22.0 & $<0.0001$ & 6.5 & $<0.0001$ & 18.0 & $<0.0001$ & 8.0 & 0.5885 \\
\hline $\mathrm{L}$ & 84 & 21.5 & $<0.0001$ & 9.0 & $<0.0001$ & 12.0 & $<0.0001$ & 9.5 & 0.023 \\
\hline $\mathrm{M}$ & 63 & 21.5 & $<0.0001$ & 13.5 & $<0.0001$ & 16.5 & $<0.0001$ & 9.5 & 0.0534 \\
\hline $\mathrm{N}$ & 139 & 21.5 & $<0.0001$ & 10.5 & $<0.0001$ & 14.5 & $<0.0001$ & 9.5 & 0.0009 \\
\hline $\mathrm{O}$ & 36 & 22.0 & $<0.0001$ & 13.0 & 0.0002 & 15.0 & $<0.0001$ & 5.0 & 0.049 \\
\hline $\mathrm{P}$ & 15 & 21.0 & 0.0151 & 16.5 & 0.0486 & 19.5 & 0.0192 & 5.5 & 0.1943 \\
\hline Q & 55 & 24.0 & $<0.0001$ & 15.5 & $<0.0001$ & 18.5 & $<0.0001$ & 7.5 & 0.1333 \\
\hline $\mathrm{R}$ & 35 & 21.5 & 0.0008 & 17.5 & 0.0003 & 16.5 & 0.0003 & 5.0 & 0.1991 \\
\hline $\mathrm{S}$ & 15 & 23.5 & 0.0093 & 18.5 & 0.0169 & 21.0 & 0.0122 & 3.5 & 0.5132 \\
\hline
\end{tabular}

Values are presented as the cut off of mortality rate of each degree in Kagoshima region (A S; supported Fig. 5B) and showed as maximum, minimum, mean temperature and temperature difference within a day (maximum temperature - minimum temperature). $p, p$-value.

\section{Figures}


A

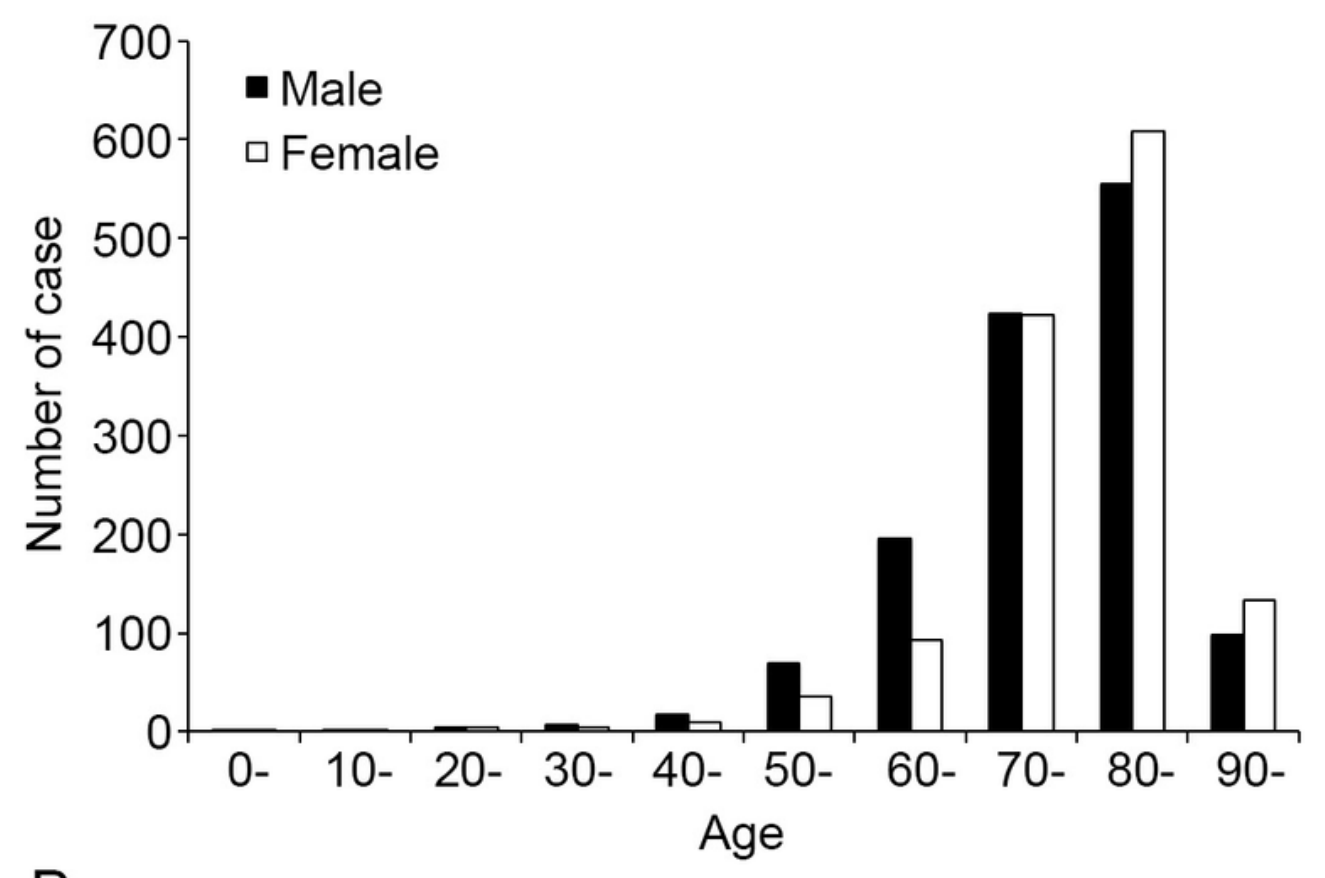

B

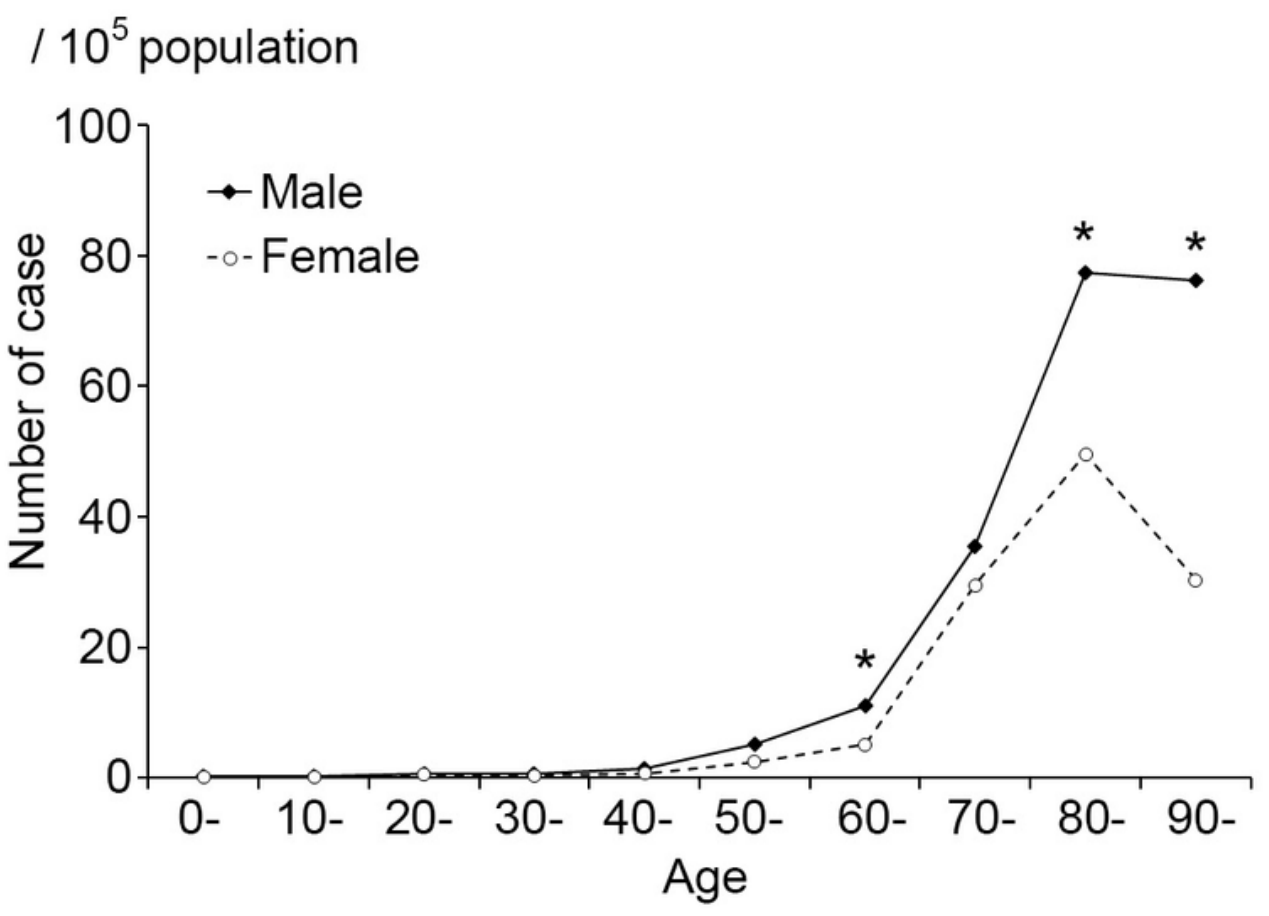

Figure 1

(A) Distribution of age and gender in bath-related death. (B) Crude mortality rates by age. The mortality rates were compared between men and women using Z-test under a binomial distribution of counts. ${ }^{*} \mathrm{p}<0.05$ men vs. women. 


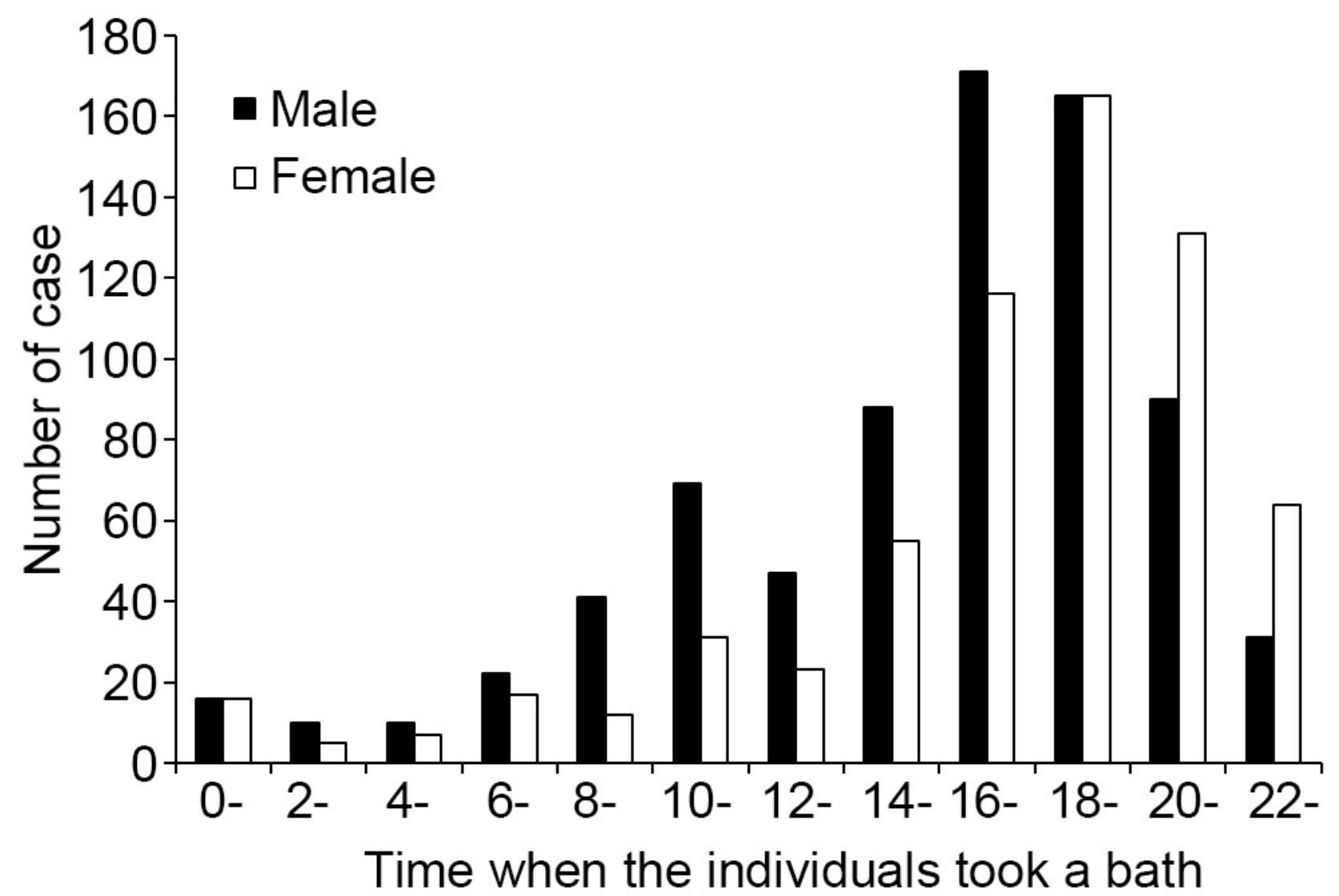

Figure 2

Time of the day when the victims with bath-related death took a bath. 


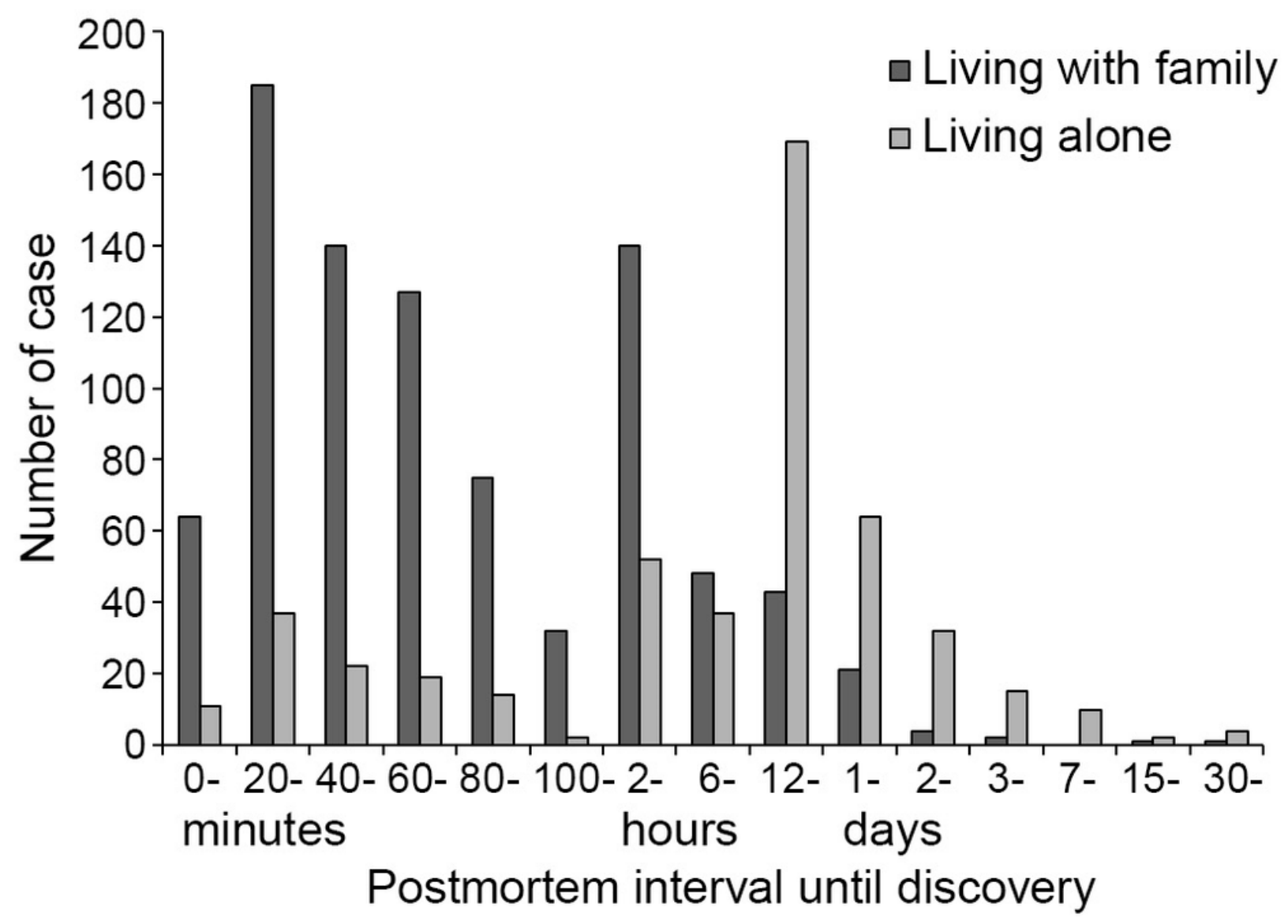

Figure 3

Postmortem interval until discovery. The black bars and the white bars indicate the postmortem interval until discovery in those who lived with their families and in those who lived alone, respectively. The data was extracted by bath-related death that occurred in home. 


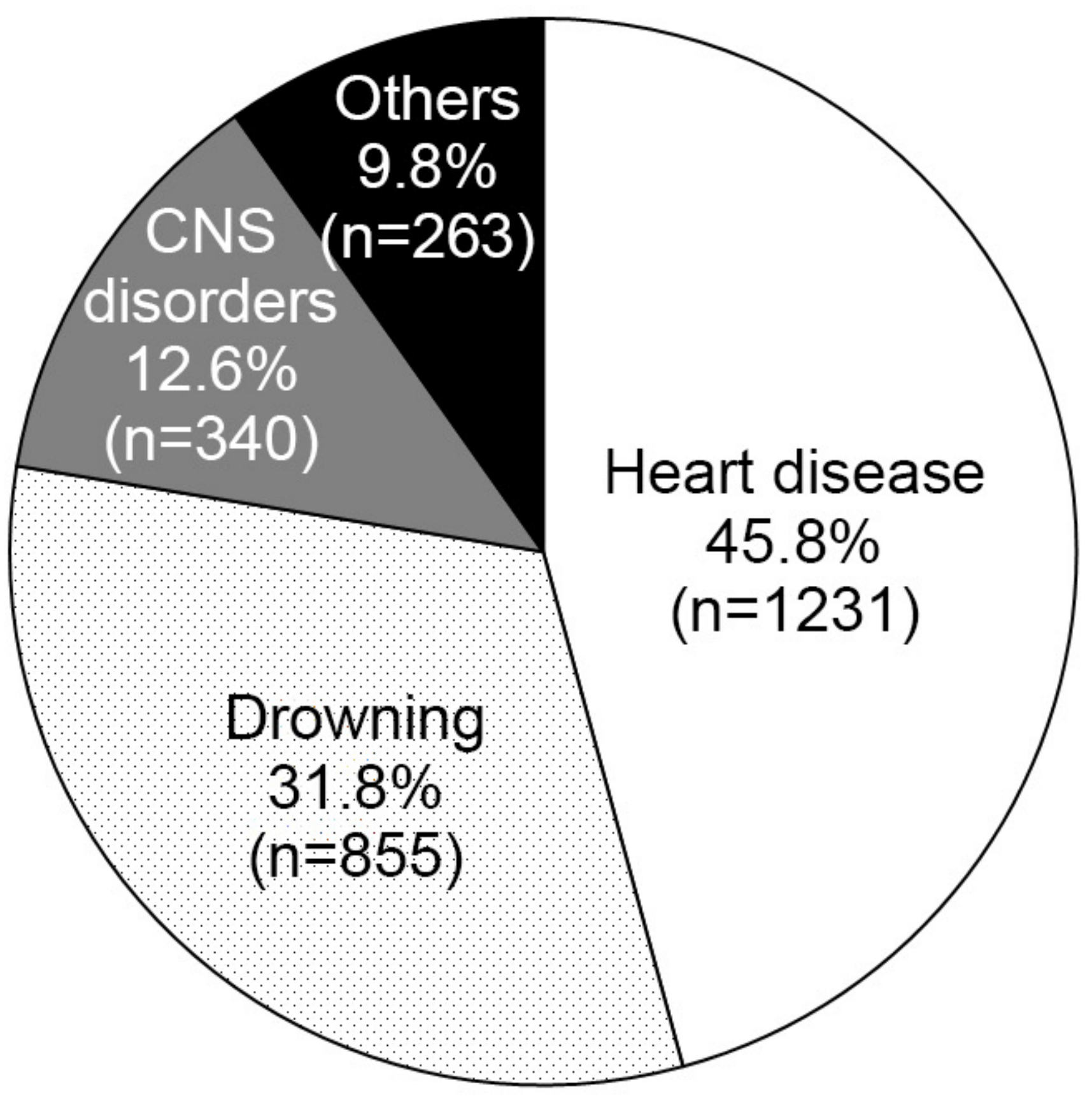

Figure 4

Cause of death on the death certificate. CNS, Central nervous system; Others including unknown. 


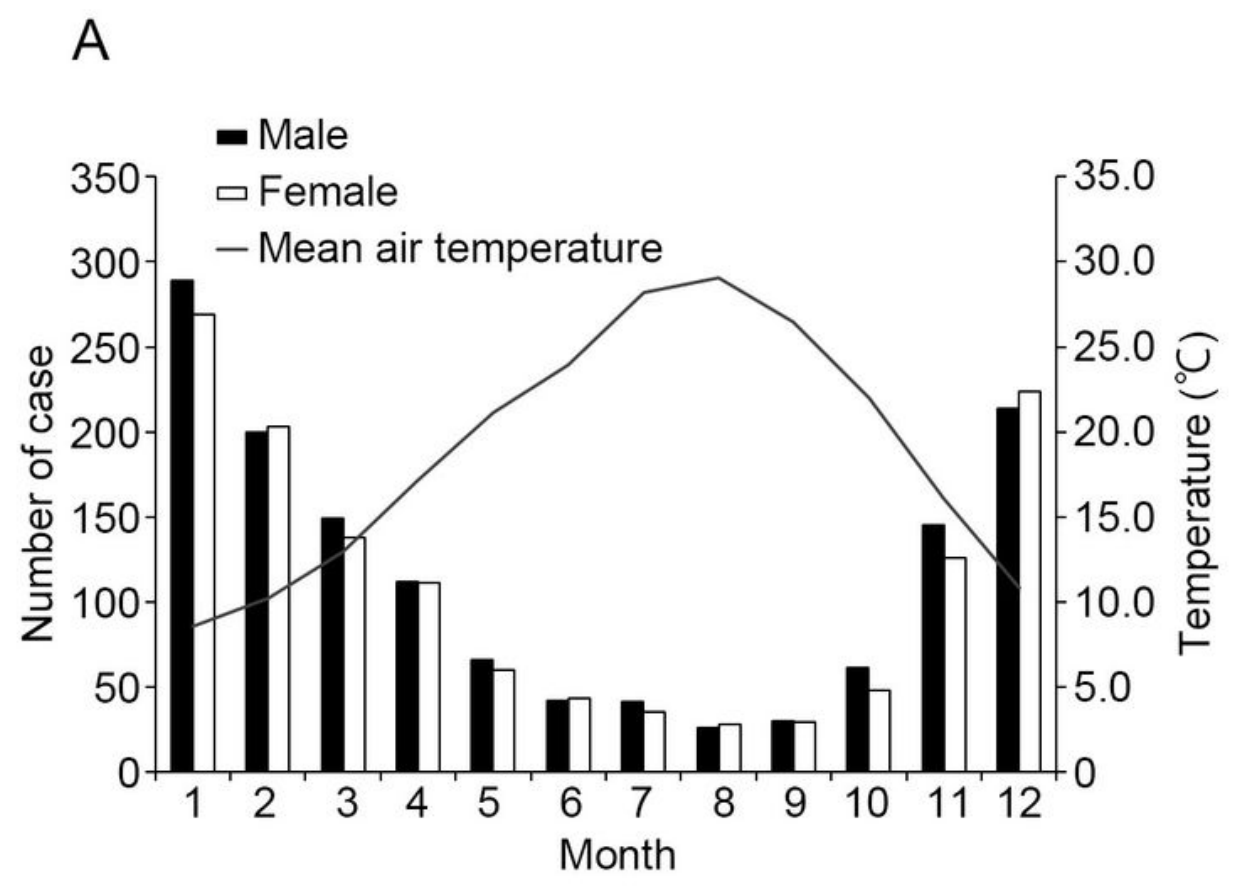

B

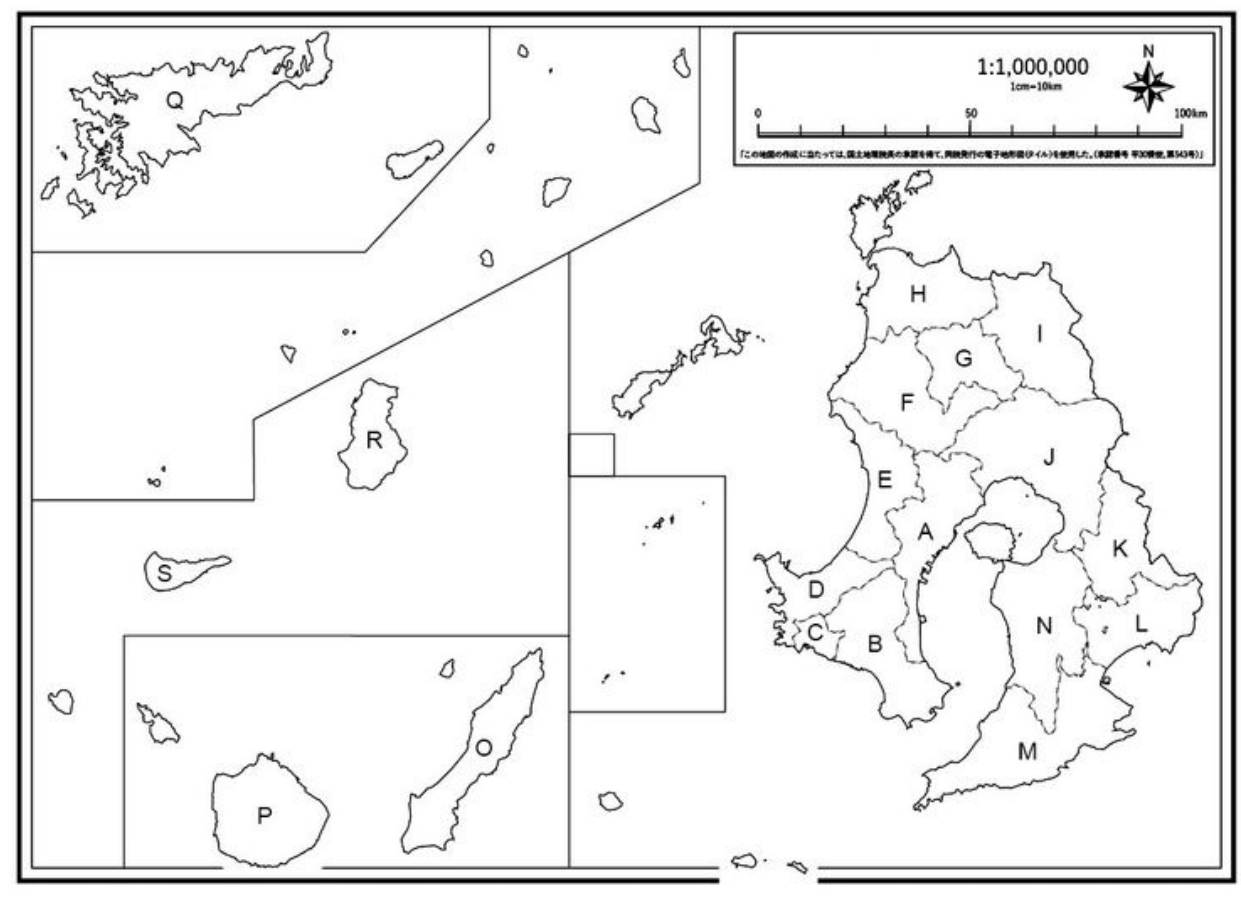

\section{Figure 5}

(A) Distribution of the number of bath-related deaths by month. The seasonal trend in the occurrence in winter (December to February). Monthly distribution of the number of bath-related death and the average daily air temperature. Fatal events were more frequent during colder months than during warmer ones. (B) The map of Kagoshima prefecture. A N, these areas are the southern tip of the main island. $0 \sim \mathrm{S}$, these areas are isolated islands. $\mathrm{O}$, the location of the nearest main island $\left(\mathrm{N} 30^{\circ} 43.0^{\prime} \mathrm{E} 130^{\circ} 58.9^{\prime}\right)$. $\mathrm{S}$, the 
location of furthermost from Kagoshima prefecture $\left(\mathrm{N} 27^{\circ} 23.2^{\prime} \mathrm{E} 128^{\circ} 39.1^{\prime}\right)$. Q, the location at the middle between $\mathrm{O}$ and $\mathrm{S}$.

\section{Supplementary Files}

This is a list of supplementary files associated with this preprint. Click to download.

- paper6.20210318SRSupplementalTable1.docx 